\title{
HILBERT SPACES OF TEMPERED DISTRIBUTIONS, HERMITE EXPANSIONS AND SEQUENCE SPACES
}

\author{
by RAINER H. PICARD
}

(Received 9th August 1989)

\begin{abstract}
Although it is well-known that tempered distributions on $\mathbb{R}^{n}$ can be expanded into series of Hermite functions, it does not seem to be known, however, that expansions of this type are accessible through the elementary concept of orthonormal expansions in Hilbert space. This approach is developed here complementing previous work on a Hilbert space approach to distributions. The basis of the development is the observation that the Hermite functions are a complete orthogonal set in each space of a certain scale of Sobolev type Hilbert spaces associated with the family of differential operators defined by
\end{abstract}

$$
\left(\mathscr{D}_{k} \Phi\right)(x)=(2 \pi i)^{-1} \exp (\pi x \cdot x) \frac{\partial}{\partial x_{k}}(\exp (-\pi x \cdot x) \Phi(x)), \quad k=1,2, \ldots, n, \quad x \in \mathbb{R}^{n} .
$$

Here $\Phi$ denotes a smooth function with compact support. The setting is first developed in the one-dimensional case. By use of the usual multi-index notation this can be extended to the higher-dimensional case. As applications various imbedding results are derived. The paper concludes with a characterization of tempered distributions by convergent Hermite expansions.

1980 Mathematics subject classification (1985 Revision): 46A12, 46E35, 42C10, 46FO5.

\section{Introduction}

In a previous study, [7], it has been demonstrated that distributions can be discussed comfortably in a Sobolev space setting. Because of the simplicity and richness of Hilbert space as a functional analytical concept it appears to facilitate the approach to distributions. This intention is common with the so-called sequential approach to distributions as developed in [3]. In fact, the above mentioned ideas complement the sequential approach by providing further insight into the structure of distributions. The basis of the investigation of distributions in $[6,7]$ is the observation that tempered distributions can be considered as elements of certain Sobolev type spaces. These are part of a chain of Hilbert spaces of which the inductive limit can be identified as the space of tempered distributions. It has been noted, [7], that Hermite functions are orthogonal in each space of the chain. The present investigation is initiated by this observation. There is a close relationship with the latter parts of [3, Chapter 8] and later, which rely heavily on Hermite expansions. We shall, however, not have to make use of the extended Köthe space theory, although interesting in its own right, developed in [3], but shall rather employ the elementary ideas of expansions with respect to 
orthonormal systems in a Hilbert space. The Hilbert space environment also provides a tight control of the relationship between elements of a Hilbert space and the sequence space of its coefficients with respect to a complete orthonormal system by an explicit unitary mapping (Parseval's equality). As a general reference for the functional analytical concepts used see e.g. $[1,2],[4]$. As a general reference for more classical material on the Fourier transform we mention [8].

\section{Sobolev type Hilbert spaces of tempered distributions}

We shall define a chain of Sobolev type Hilbert spaces based not on the usual concept of a derivative but on a modified differentiation operator related to the tempered derivative, (see [3, p. 161]. As a brief introduction into chains of Hilbert spaces see [5, Chapter VI]). For convenience we shall initially consider only the onedimensional case and comment on the higher-dimensional analogues in a later section. In the definition of these Sobolev type spaces the Gaussian distribution function

$$
\gamma(x)=e^{-\pi x^{2}}, \quad x \in \mathbb{R}
$$

is involved. We shall follow basically the approach outlined in [6, 1.1.2.2].

We define for $\Phi \in \mathcal{C}_{\infty}(\mathbb{R})\left(\check{C}_{\infty}(\mathbb{R})\right.$ denotes the set of arbitrarily often continuously differentiable (complex-valued) functions with compact support in $\mathbb{R}$ ),

$$
\mathscr{D} \Phi:=\left(\gamma^{-1} D \gamma\right) \Phi \quad \text { for } \quad \Phi \in \mathcal{C}_{\infty}(\mathbb{R})
$$

Here

$$
\begin{gathered}
\gamma^{-1}(x):=1 / \gamma(x), \quad x \in \mathbb{R}, \\
D \Phi=(2 \pi i)^{-1} d \Phi \quad \text { for } \quad \Phi \in \mathcal{C}_{\infty}(\mathbb{R}),
\end{gathered}
$$

where the " $d$ " indicates ordinary differentiation with respect to the argument. Denoting the Fourier transform by $\mathscr{F}$ with the convention that

$$
(\mathscr{F} \Phi)(x)=\int_{\mathbf{R}} e^{-\pi i x t} \Phi(t) \mathrm{dt} \text { for all } \Phi \in \dot{C}_{\infty}(\mathbb{R}),
$$

we have that $\mathscr{F}$ is a spectral representation for the operator $\underline{D}$, where $\underline{D}$ denotes the closure of $D$, i.e.

$$
(\mathscr{F} \underline{D} \Phi)(x)=x(\mathscr{F} \Phi)(x), \quad x \in \mathbb{R} .
$$

If we represent the self-adjoint extension of the "multiplication-by-the-argument' operator in $L_{2}(\mathbb{R})$ by $m$, then we write this as

$$
\mathscr{F} \mathscr{Q}=m \mathscr{F} \quad \text { on } \quad \dot{C}_{\infty}(\mathbb{R})
$$


Making use of the knowledge that the inverse Fourier transform $\mathscr{F}^{*}$ is very similar to $\mathscr{F}$ we also have

$$
\mathscr{F}_{\underline{m}}=-\underline{D} \mathscr{F} \quad \text { on } \quad \mathcal{C}_{\infty}(\mathbb{R})
$$

Noting that

$$
\underline{D}=\underline{D}+i \underline{m},
$$

this implies

$$
i \mathscr{F} \mathscr{D}=\mathscr{Q} \mathscr{F} \quad \text { on } \quad \mathcal{C}_{\infty}(\mathbb{R})
$$

Clearly, the adjoint $\mathscr{Q}^{*}$ of $\mathscr{D}$ satisfies

$$
\mathscr{Q}^{*} \Phi=\gamma \underline{D} \gamma^{-1} \Phi \equiv(\underline{D}-i \underline{m}) \Phi, \quad \Phi \in \mathcal{C}_{\infty}(\mathbb{R})
$$

The operator $\mathscr{Q}^{*}$ is known as the tempered derivative (see $\left.[3, \mathrm{p} .161]\right)$.

To develop a proper setting for the operator $\mathscr{D}$ we start with the observation that for $\Phi \in \mathcal{C}_{\infty}(\mathbb{R})$ we have

$$
\begin{aligned}
\|\underline{\mathscr{D}} \Phi\|^{2} & =\|(\underline{D}+i \underline{m}) \Phi\|^{2}=\|\underline{D} \Phi\|^{2}+\|\underline{m} \Phi\|^{2}+2 \operatorname{Re}(i(\underline{D} \Phi, \underline{m} \Phi)) \\
& =\|\underline{D} \Phi\|^{2}+\|\underline{m} \Phi\|^{2}-\frac{1}{\pi} \operatorname{Re}(d \Phi, \underline{m} \Phi) \\
& =\|\underline{D} \Phi\|^{2}+\|\underline{m} \Phi\|^{2}-\frac{1}{\pi} \int \frac{1}{2} t \frac{d}{d t}|\Phi(t)|^{2} d t \\
& =\|\underline{D} \Phi\|^{2}+\|\underline{m} \Phi\|^{2}+\frac{1}{2 \pi}\|\Phi\|^{2}
\end{aligned}
$$

where $\|\cdot\|$ denotes the $L_{2}(\mathbb{R})$-norm induced by the $L_{2}(\mathbb{R})$ inner product $(\cdot, \cdot)$ (assumed to be linear in the second factor). We proceed by defining Sobolev type spaces $\mathscr{S}_{k}$ by completing $\mathcal{C}_{\infty}(\mathbb{R})$ with respect to the norm $\|\cdot\|_{\mathscr{S}, k}$ induced by the inner product

$$
(\cdot, \cdot)_{\mathscr{S}, k}=\left(\mathscr{Q}^{k} \cdot \mathscr{Q}^{k} \cdot\right)
$$

Here $k$ can be any non-negative integer, i.e. $k \in \mathbb{N}=\{0,1,2, \ldots)$, and by convention we have $\mathscr{D}^{0} \phi \equiv \phi$ for $\phi \in L_{2}(\mathbb{R})$. In particular we have

$$
\mathscr{S}_{0}=L_{2}(\mathbb{R})
$$

We note that (see $[6$, p. 27]) 


$$
D(\mathscr{D})=D\left(\mathscr{D}^{*}\right)=\mathscr{S}_{1}
$$

where $D(* *)$ denotes the domain of ${ }_{* *}$. We shall now define the Hermite functions $H_{s}$ by

$$
H_{s}=\mathscr{Q}^{s} \gamma, \quad s \in \mathbb{N}
$$

As can be easily seen by induction

$$
H_{s}=(2 i)^{s} P_{s} \gamma
$$

where $P_{s}$ is a polynomial of degree $s$ with leading coefficient 1 . We note that

$$
\mathscr{Q} * \gamma=0
$$

The connection between the functions $H_{s}$ and the Hermite polynomials $\mathscr{H}_{s}$ or alternative Hermite polynomials $\mathscr{H} e_{s}$ is

$$
\begin{aligned}
\mathscr{H}_{s}(x) & =(-i \sqrt{2 \pi})^{s} \exp \left(x^{2} / 2\right) H_{s}(x / \sqrt{2 \pi}), \\
& =(2 \sqrt{2 \pi})^{s} P_{s}(x / \sqrt{2 \pi}),
\end{aligned}
$$

and

$$
\begin{aligned}
\mathscr{H} e_{s}(x) & =(-i \sqrt{\pi})^{2} \exp \left(x^{2}\right) H_{s}(x /(2 \sqrt{\pi})), \\
& =(2 \sqrt{\pi})^{s} P_{s}(x /(2 \sqrt{\pi})), \quad x \in \mathbb{R} .
\end{aligned}
$$

The operators $\mathscr{D}$ and $\mathscr{D}^{*}$ satisfy the following commutator relation

$$
\mathscr{Q}^{*} \mathscr{Q}-\mathscr{Q} \mathscr{Q}^{*}=\pi^{-1} \text { on } \quad \mathscr{S}_{2}
$$

From (1.2), (1.8) and (1.9) we obtain our first lemma.

Lemma 1.1. We have

$$
\left(\mathscr{Q}^{*}\right)^{k} \mathscr{Q}^{k} H_{s}=\frac{(k+s) !}{s ! \pi^{k}} H_{s}
$$

and

$$
\mathscr{F} H_{s}=(-i)^{s} H_{s}, \quad \mathscr{Q}^{*} H_{s+1}=(s+1) \pi^{-1} H_{s}, \quad \mathscr{Q} H_{s}=H_{s+1},
$$

for all $k, s \in \mathbb{N}$.

Proof. The first equality of (1.11) is an immediate consequence of (1.2). The last equality in (1.11) follows directly from the definition of $H_{s}$. To see the remaining equality we only have to make use of (1.8) and (1.9). By induction we obtain 


$$
\underline{\mathscr{Q}}^{*} \underline{\mathscr{D}}^{s+1} \gamma=(s+1) \pi^{-1} \underline{\mathscr{D}}^{s} \gamma
$$

Indeed,

$$
\begin{aligned}
\mathscr{Q}^{*} \mathscr{Q}^{s+2} \gamma & =\mathscr{D}^{*} \mathscr{D}_{\mathscr{D}}^{s+1} \gamma, \\
& =\mathscr{Q} \mathscr{D} * \mathscr{Q}^{s+1} \gamma+\pi^{-1} \mathscr{\mathscr { Q }}^{s+1} \gamma, \\
& =\mathscr{D}\left((s+1) \pi^{-1} \mathscr{Q}^{s} \gamma\right)+\pi^{-1} \underline{\mathscr{Q}}^{s+1} \gamma, \\
& =(s+2) \pi^{-1} \underline{\mathscr{Q}}^{s+1} \gamma,
\end{aligned}
$$

where the induction hypothesis has been used for the third equality sign. Equality (1.10) now follows by another induction with respect to $k$ from (1.9) and (1.12).

Remark 1. Noting that $\gamma \in \mathscr{S}_{k}$ for all $k \in \mathbb{N}$ we see that the last lemma shows that the Hermite functions are eigenfunctions of the $L_{2}(\mathbb{R})$-selfadjoint operators $\left(\mathscr{D}^{*}\right)^{s} \mathscr{D}^{s}$ for any $s \in \mathbb{N}$, and also for the Fourier transform $\mathscr{F}: L_{2}(\mathbb{R}) \rightarrow L_{2}(\mathbb{R})$. Moreover, we know that $\left(H_{s}\right)_{s \in \mathbb{N}}$ is complete in $L_{2}(\mathbb{R})$.

From the completeness of $\left(H_{s}\right)_{s \in \mathbb{N}}$ in $L_{2}(\mathbb{R})$ we obtain its completeness in $\mathscr{S}_{k}, k \in \mathbb{N}$.

Lemma 1.2. Let $p, m \in \mathbb{N}$ and $p \leqq m$. Then we have that the Hilbert space $\mathscr{S}_{m}$ is continuously imbedded into $\mathscr{S}_{p}$, i.e. $\mathscr{S}_{m} \hookrightarrow \mathscr{S}_{p}$. In particular, we have

$$
\|\Phi\|_{\mathscr{S}, p}^{2} \leqq(2 \pi)^{m-p}\|\Phi\|_{\mathscr{S}^{, m}}^{2} \quad \text { for } \quad \Phi \in \mathscr{S}_{m}
$$

The family $\left(H_{s}\right)_{s \in \mathbb{N}}$ is complete and orthogonal in $\mathscr{S}_{k}$ for any $k \in \mathbb{N}$. We have

$$
\left\|H_{s}\right\|_{\mathscr{S}, k}^{2}=2^{-1 / 2}(k+s) ! \pi^{-(k+s)}
$$

for $s, k \in \mathbb{N}$.

Proof. The imbedding result follows by induction, [6, Lemma 1.7]. We have

$$
\begin{aligned}
\left\|\mathscr{D}^{k+1} \Phi\right\|^{2} & =\left\|(\mathscr{D}+i m) \mathscr{D}^{k} \Phi\right\|^{2} \\
& =\left\|\underline{D} \underline{\mathscr{Q}}^{k} \Phi\right\|^{2}+\left\|\underline{\underline{m} \mathscr{D}}{ }^{k} \Phi\right\|^{2}+\frac{1}{2 \pi}\left\|\mathscr{D}^{k} \Phi\right\|^{2}
\end{aligned}
$$

Thus the continuous imbedding is clear by induction. The orthogonality of $\left(H_{s}\right)_{s \in \mathbb{N}}$ in $\mathscr{S}_{k}$ follows by a similarly elementary calculation. Let $s \leqq t, s, t \in \mathbb{N}$, then

$$
\left(H_{s}, H_{t}\right)_{\mathscr{S}, k}=\left(\mathscr{Q}^{s+k} \gamma, \mathscr{Q}^{t+k} \gamma\right)
$$




$$
\begin{aligned}
& =\left(\left(\mathscr{Q}^{*}\right)^{t+k} \mathscr{D}^{s+k} \gamma, \gamma\right), \\
& =\left(\left(\mathscr{Q}^{*}\right)^{t-s}\left(\mathscr{Q}^{*}\right)^{s+k} \gamma, \gamma\right), \\
& =\frac{(k+s) !}{\pi^{s+k}}\left(\left(\mathscr{Q}^{*}\right)^{t-s} \gamma, \gamma\right),
\end{aligned}
$$

where the last equality is a consequence of Lemma 1.1. By (1.8) the orthogonality now follows and for $s=t$ using

$$
\int_{\mathbb{R}} \gamma(t)^{2} d t=2^{-1 / 2}
$$

we also get (1.14). To see completeness let us assume there is an $f \in \mathscr{S}_{k}, k \in \mathbb{N}$ fixed, such that

$$
\left(f, H_{s}\right)_{S_{, k}}=0 \text { for all } s \in \mathbb{N}
$$

Then we have to show that $f$ can only be the zero element. We have

$$
\begin{aligned}
\left(f, H_{s}\right)_{\mathscr{S}, k} & =\left(\mathscr{Q}^{k} f, \mathscr{Q}^{k} H_{s}\right)=\left(f,\left(\mathscr{Q}^{*}\right)^{k} \mathscr{Q}^{k} H_{s}\right) \\
& =\frac{(k+s) !}{s ! \pi^{k}}\left(f, H_{s}\right) .
\end{aligned}
$$

From (1.16) we see that indeed $f \equiv 0$ now follows from the completeness of the Hermite functions in $L_{2}(\mathbb{R})$.

We are now ready to establish the corresponding expansions with respect to $\left(H_{s}\right)_{s \in \mathrm{N}}$. For this we normalize $\left(H_{s}\right)_{s \in N}$ for each particular $\mathscr{S}_{k}$, we define in accordance with Lemma 1.1

$$
\mathscr{H}_{s}^{k}=2^{1 / 4} \pi^{(k+s) / 2} / \sqrt{(k+s) !} H_{s}=\pi^{k / 2} \sqrt{s ! /(k+s) !} \mathscr{H}_{s}^{0}
$$

for $s, k \in \mathbb{N}$.

Note in particular, that

$$
\begin{gathered}
\mathscr{H}_{s}^{0}=2^{1 / 4} \pi^{s / 2} / \sqrt{s !} H_{s}, \\
\mathscr{Q} \mathscr{H}_{s}^{0}=\pi^{-1 / 2} \sqrt{s+1} \mathscr{H}_{s+1}^{0} \\
\mathscr{Q}^{*} \mathscr{H}_{s+1}^{0}=\pi^{-1 / 2} \sqrt{s+1} \mathscr{H}_{s}^{0}, \quad s \in \mathbb{N} .
\end{gathered}
$$


For any $f \in \mathscr{S}_{k}$ we have now an expansion w.r.t. the orthonormal system $\left(\mathscr{H}_{s}^{k}\right)_{s \in N}$

$$
f=\sum_{s \in N} \alpha_{s}^{k}(f) \mathscr{H}_{s}^{k}
$$

such that

$$
\|f\|_{\mathcal{S}, k}=\sqrt{\sum_{s \in \mathbf{N}}\left|\alpha_{s}^{k}(f)\right|^{2}} .
$$

Since, however, $\mathscr{S}_{k}$ is continuously imbedded into $\mathscr{P}_{k-j}$ for $j=0,1,2, \ldots, k$, we also have expansions in these other spaces for $f \in \mathscr{S}_{k}$. We shall now establish the relationship between the respective coefficients.

Lemma 1.3. For the coefficient $\alpha_{s}^{j}(f), s \in \mathbb{N}, j=0,1,2, \ldots, k$, of a given element $f \in \mathscr{S}_{k}$ we have the following relationship

$$
\alpha_{s}^{j}(f)=\pi^{-j / 2} \sqrt{(j+s) ! / s !} \alpha_{s}^{0}(f) .
$$

Proof. The result follows straightforwardly. We have

$$
\begin{aligned}
& \alpha_{s}^{j}(f)=\left(\mathscr{H}_{s}^{j}, f\right)_{\mathscr{S}, j}=2^{1 / 4} \pi^{(j+s) / 2} / \sqrt{(j+s) !}\left(H_{s}, f\right)_{\mathscr{S}, j} \\
& =2^{1 / 4} \pi^{(j+s) / 2} / \sqrt{(j+s) !}\left(\mathscr{Q}^{j} H_{s}, \mathscr{Q}^{j} f\right) \\
& =2^{1 / 4} \pi^{(j+s) / 2} / \sqrt{(j+s) !}\left(\left(\mathscr{D}^{*}\right)^{j} \mathscr{D}^{j} H_{s}, f\right) \\
& =2^{1 / 4} \pi^{(j+s) / 2} / \sqrt{(j+s) !} \frac{(j+s) !}{s ! \pi^{j}}\left(H_{s}, f\right) \\
& =2^{1 / 4} \pi^{-j / 2} \pi^{s / 2} \sqrt{(j+s) !} \frac{1}{s !}\left(H_{s}, f\right) \\
& =\pi^{-j / 2} \sqrt{(j+s) ! / s !}\left(\mathscr{H}_{s}^{0}, f\right) \\
& =\pi^{-j / 2} \sqrt{(j+s) ! / s !} \alpha_{0}^{s}(f) .
\end{aligned}
$$

Defining now sequence Hilbert spaces $\partial_{k}$ as the completion of eventually vanishing sequences $\left(\alpha_{s}\right)_{s \in N}$ (i.e. $\alpha_{s} \equiv 0$ for all sufficiently large $s$ ) relative to the norm $\|\cdot\|_{o, k}$ induced by the inner product 


$$
(\alpha, \beta)_{0, k}=\pi^{-k} \sum_{s \in \mathbb{N}}(k+s) ! / s ! \bar{\alpha}_{s} \beta_{s}, \quad \alpha=\left(\alpha_{s}\right)_{s}, \quad \beta=\left(\beta_{s}\right)_{s} \in J_{k} .
$$

we can formulate our first theorem. For convenience we shall assume from now on that $\alpha_{s} \equiv 0$ for $s<0$.

Theorem 1. The spaces $\mathscr{S}_{k}$ and $\jmath_{k}$ are unitarily equivalent. The unitary mapping, $\mathscr{Y}$ between them is given by

$$
\begin{gathered}
\mathscr{Y}: \mathscr{Y}_{k} \rightarrow J_{k} \\
f \rightarrow\left(\left(\mathscr{H}_{s}^{0}, f\right)\right)_{s} \equiv\left(\alpha_{s}^{0}(f)\right)_{s} .
\end{gathered}
$$

In particular, we have

$$
\|f\|_{\mathscr{S}, k}=\|\mathscr{Y} f\|_{o, k}=\pi^{-k / 2} \sqrt{\sum_{s \in \mathbb{N}}(k+s) ! / s !\left|\left(\mathscr{H}_{s}^{0}, f\right)\right|^{2}},
$$

for all $f \in \mathscr{P}_{k}, k \in \mathbb{N}$. The inverse $\mathscr{Y}^{*}$ of $\mathscr{Y}$ is given by

$$
\begin{gathered}
\mathscr{Y}^{*}: \unlhd_{k} \rightarrow \mathscr{Y}_{k} \\
\alpha=\left(\alpha_{s}\right)_{s} \rightarrow \sum_{s \in N} \alpha_{s} \mathscr{H}_{s}^{0} .
\end{gathered}
$$

The operations $\mathscr{Q}, \mathscr{F}$ and their adjoints are transferred by $\mathscr{Y}$ to corresponding operations in $\unlhd_{k}, k \in \mathbb{N}$. We have for all $\alpha \in \bigcup_{k \geqq 1} \supset_{k}$

$$
\mathscr{Y} \mathscr{D} \mathscr{Y}^{*} \alpha=\left(\pi^{1 / 2} \sqrt{s} \alpha_{s-1}\right)_{s}, \mathscr{Y} \mathscr{Q}^{*} \mathscr{O} * \alpha=\left(\pi^{-1 / 2} \sqrt{s+1} \alpha_{s+1}\right)_{s}
$$

and

$$
\mathscr{Y} \mathscr{F} \mathscr{Y}^{*} \alpha=\left((-i)^{s} \alpha_{s}\right)_{s}, \quad \mathscr{Y} \mathscr{F} * \mathscr{Y}^{*} \alpha=\left(i^{s} \alpha_{s}\right)_{s}
$$

for all $\alpha \in \bigcup_{k \in N} \jmath_{k}$.

Proof. The mapping $\mathscr{Y}$ associates with any $f \in \mathscr{P}_{k}$ its sequence of coefficients in the expansion of $f$ with respect to $\left(\mathscr{H}_{s}^{0}\right)_{s}$ in $\mathscr{S}_{0}$. That $\mathscr{Y}$ is an isometry should be clear according to Lemma 1.3. To see that $\mathscr{Y}$ is also onto we notice that $\diamond_{k}$ is obviously continuously imbedded in $\varsigma_{0}$, in fact,

$$
\|\alpha\|_{0,0} \leqq \pi^{k / 2}\|\alpha\|_{0, k} \text { for all } \alpha \in \searrow_{k},
$$

so that $f:=\sum_{s \in N} \alpha_{s} \mathscr{H}_{s}^{0}$ is a well-defined element in $\mathscr{S}_{0} \equiv L_{2}(\mathbb{R})$ with

$$
\alpha_{s}=\alpha_{s}^{0}(f)
$$


Since $\alpha \in \Im_{k}$ we also have by the isometry property of $\mathscr{Y}$ that $\sum_{s \in N} \alpha_{s} \mathscr{H}_{s}^{0}$ converges in $\mathscr{S}_{k}$. The limit in $\mathscr{S}_{k}$ must coincide with $f$ by the continuity of the imbedding of $\mathscr{S}_{k}$ in $L_{2}(\mathbb{R})$, i.e. $f \in \mathscr{S}_{k}$.

This proves that $\mathscr{Y}$ is onto and that $\mathscr{Y}^{*}$ has the form (1.23). To establish the transfer rules (1.24), (1.25) we note that by (1.18)

$$
\mathscr{D} \sum_{s \in N} \alpha_{s} \mathscr{H}_{s}^{0}=\sum_{s \in N} \alpha_{s-1} \pi^{-1 / 2} \sqrt{s} \mathscr{H}_{s}^{0},
$$

and

$$
\mathscr{Q}^{*} \sum_{s \in \mathbb{N}} \alpha_{s} \mathscr{H}_{s}^{0}=\sum_{s \in \mathbb{N}} \alpha_{s+1} \pi^{-1 / 2} \sqrt{s+1} \mathscr{H}_{s}^{0} .
$$

Equalities (1.25) follow immediately from (1.11):

$$
\mathscr{F} \sum_{s \in \mathbb{N}} \alpha_{s} \mathscr{H}_{s}^{0}=\sum_{s \in \mathbb{N}} \alpha_{s}(-i)^{s} \mathscr{H}_{s}^{0}, \quad \mathscr{F}^{*} \sum_{s \in \mathrm{N}} \alpha_{s} \mathscr{H}_{s}^{0}=\sum_{s \in \mathbb{N}} \alpha_{s} i^{s} \mathscr{H}_{s}^{0} .
$$

Remark 2. It is interesting to note that $\mathscr{Y}$ is actually independent of $k \in \mathbb{N}$, a fact that has already been expressed by not indicating to what domain $\mathscr{Y}$ is applied. So, we may consider $\mathscr{Y}$ as acting on $\mathscr{S}_{0}$ and the criterion for $f$ being in $\mathscr{S}_{k}$ is if $\mathscr{Y}_{f}$ is a sequence of coefficients that is bounded in the norm of $\jmath_{k}$.

We shall now extend our considerations to the space $\mathscr{S}_{-k}$ of distributions over $\mathscr{S}_{k}$ equipped with the usual functional norm

$$
\|g\|_{\mathcal{S}_{,-k}:=} \sup _{\phi \in \mathscr{S}_{k}-\{0\}} \frac{|g(\phi)|}{\|\phi\|_{\mathscr{S}_{, k}}}
$$

for $g \in \mathscr{S}_{-k}, k \in \mathbb{N}$. The Riesz representation theorem yields the existence of an isometric isomorphism

$$
R_{k}: \mathscr{S}_{-k} \rightarrow \mathscr{S}_{k},
$$

with $f(\phi)=\left(R_{k} f, \phi\right)_{\mathscr{S}_{, k}}$ for all $\phi \in \mathscr{S}_{k}, f \in \mathscr{S}_{-k}$. $R_{k}$ can be used to introduce a Hilbert space structure in $\mathscr{S}_{-k}$ by letting

$$
(f, g)_{\mathscr{Y}_{,-k}:}:=\left(R_{k} f, R_{k} g\right)_{\mathscr{Y}, k}, \quad f, g \in \mathscr{S}_{-k} .
$$

Thus $R_{k}$ becomes a unitary map between $\mathscr{S}_{-k}$ and $\mathscr{S}_{k}$. In particular,

$$
\left\|R_{k} f\right\|_{\mathscr{S}, k}=\sup _{\left.\phi \in \mathscr{S}_{k}-10\right\}} \frac{\left|\left(R_{k} f, \phi\right)\right|}{\|\phi\|_{\mathscr{S}, k}}=\sup _{\phi \in \mathscr{S}_{k}-\{0)} \frac{|f(\phi)|}{\|\phi\|_{\mathscr{S}, k}}=\|f\|_{\mathcal{S}_{,-k} .}
$$


Since $R_{0}$ is the identity it seems suggestive to introduce as a notation

$$
(f, \phi):=f(\phi),
$$

and

$$
(\phi, f):=\overline{(f, \phi)} \text { for } f \in \mathscr{S}_{-k}, \quad \phi \in \mathscr{S}_{k} .
$$

We can now extend the meaning of

$$
\mathscr{Q}: \mathscr{S}_{k+1} \rightarrow \mathscr{S}_{k}, \mathscr{Q}^{*}: \mathscr{S}_{k+1} \rightarrow \mathscr{S}_{k}, \mathscr{F}: \mathscr{S}_{k} \rightarrow \mathscr{S}_{k}, \mathscr{F}^{*}: \mathscr{S}_{k} \rightarrow \mathscr{S}_{k}
$$

initially defined for $k \in \mathbb{N}$ to negative indices in the usual way. Let

$$
\begin{gathered}
(\mathscr{D} f, \Phi):=\left(f, \mathscr{D}^{*} \Phi\right) \text { for } f \in \mathscr{S}_{-k} \text { and all } \Phi \in \mathscr{S}_{k+1}, \\
\left(\mathscr{D}^{*} f, \Phi\right):=(f, \mathscr{D} \Phi) \text { for } f \in \mathscr{S}_{-k} \text { and all } \Phi \in \mathscr{S}_{k+1}, \\
(\mathscr{F} f, \Phi):=\left(f, \mathscr{F}^{*} \Phi\right) \text { for } f \in \mathscr{S}_{-k} \text { and all } \Phi \in \mathscr{S}_{k}, \\
\left(\mathscr{F}^{*} f, \Phi\right):=(f, \mathscr{F} \Phi) \text { for } f \in \mathscr{S}_{-k} \text { and all } \Phi \in \mathscr{Y}_{k},
\end{gathered}
$$

for $k \in \mathbb{N}$. As an immediate consequence of these definitions we obtain by duality arguments the following extension of Lemma 1.2.

Lemma 1.4. Let $p, m \in \mathbb{Z}$ and $p \leqq m$. Then we have that the Hilbert space $\mathscr{S}_{m}$ is continuously imbedded into $\mathscr{S}_{p}$, i.e. $\mathscr{S}_{m} \hookrightarrow \mathscr{S}_{p}$. In particular, we have

$$
\|\Phi\|_{\mathscr{S}, p}^{2} \leqq(2 \pi)^{m-p}\|\Phi\|_{\mathscr{S}, m}^{2} \text { for } \Phi \in \mathscr{S}_{m}
$$

The family $\left(\mathscr{H}_{s}^{k}\right)_{s \in \mathrm{N}}$, with $\mathscr{H}_{s}^{k}=\pi^{k-2} \sqrt{s ! /(|k|+s) ! !^{\mathrm{gn}(k)}} \mathscr{H}_{s}^{0}$, is complete and orthonormal in $\mathscr{S}_{k}$ for any $k \in \mathbb{Z}$. We have in particular

$$
\left\|\mathscr{H}_{s}^{0}\right\|_{\mathscr{S}^{2}, k}^{2}=\left\{\frac{(|k|+s) !}{s ! \pi^{|k|}}\right\}^{\operatorname{sgn}(k)}
$$

for $s \in \mathbb{N}, k \in \mathbb{Z}$. For $k \in \mathbb{N}$ the Riesz map $R_{k}: \mathscr{S}_{-k} \rightarrow \mathscr{S}_{k}$ is the inverse of $\left(\mathscr{Q}^{*}\right)^{k} \mathscr{Q}^{k}: \mathscr{S}_{k} \rightarrow \mathscr{S}_{-k}$.

Proof. We shall only indicate the reasoning since all statements follow by standard duality arguments. To see (1.31) we may w.l.o.g. assume that $m \leqq 0$ and argue as follows. Let $\Phi \in \mathscr{S}_{m}$ then by using Lemma $1.2(-p \geqq-m \geqq 0)$

$$
\begin{aligned}
\|\Phi\|_{\mathscr{Y}, p} & =\sup _{\phi \in \mathscr{S}_{-p}-\{0\}} \frac{|\Phi(\phi)|}{\|\phi\|_{\mathscr{S}_{,-p}}} \leqq(2 \pi)^{(m-p) / 2} \sup _{\phi \in \mathscr{S}_{-m}-\{0)} \frac{|\Phi(\phi)|}{\|\phi\|_{\mathscr{S}_{,-m}}} \\
& =(2 \pi)^{(m-p) / 2}\|\Phi\|_{\mathscr{S}_{, m} .}
\end{aligned}
$$

For the remainder of the Lemma we observe that by Lemma 1.1 and the above definitions $(k \in \mathbb{N})$ 


$$
\begin{aligned}
\left(R_{k} \mathscr{H}_{s}^{k}, \phi\right)_{\mathscr{S}, k} \equiv\left(\mathscr{H}_{s}^{k}, \phi\right) & =\frac{s ! \pi^{k}}{(k+s) !}\left(\left(\mathscr{Q}^{*}\right)^{k} \mathscr{Q}^{k} \mathscr{H}_{s}^{k}, \phi\right) \\
& =\frac{s ! \pi^{k}}{(k+s) !}\left(\mathscr{D}^{k} \mathscr{H}_{s}^{k}, \mathscr{D}^{k} \phi\right)=\frac{s ! \pi^{k}}{(k+s) !}\left(\mathscr{H}_{s}^{k}, \phi\right)_{\mathscr{S}_{, k}},
\end{aligned}
$$

for all $\phi \in \mathcal{C}_{\infty}^{2}(\mathbb{R})$, and therefore

$$
R_{\mathrm{k}} \mathscr{H}_{s}^{k}=\frac{s ! \pi^{k}}{(k+s) !} \mathscr{H}_{s}^{k}
$$

From this we obtain immediately (1.32) as well as the completeness and orthonormality statement. The last statement of the lemma is clear from the following sequence of equalities utilizing the above definitions and has been included for later reference:

$$
(f, \phi)=\left(R_{k} f, \phi\right)_{\mathcal{S}, k}=\left(\mathscr{D}^{k} R_{k} f, \mathscr{D}^{k} \phi\right)=\left(\left(\mathscr{D}^{*}\right)^{k} \mathscr{D}^{k} R_{k} f, \phi\right)
$$

The mapping $\mathscr{Y}$ can now also be extended in a natural way to distributions by associating

$$
f \rightarrow\left(\left(\mathscr{H}_{s}^{0}, f\right)\right)_{s}
$$

where now $k$ can be any integer, i.e. $k \in \mathbb{Z}$. We still have to determine a suitable sequence space to maintain the isometric character of $\mathscr{Y}$. Using the Riesz map we see that by repeated application of $(1.18)(k \in \mathbb{N})$

$$
\begin{aligned}
\left|\left(f, \mathscr{H}_{s}^{0}\right)\right| & =\left|\left(R_{k} f, \mathscr{H}_{s}^{0}\right)_{\mathscr{S}, k}\right| \\
& =\pi^{-k / 2} \sqrt{(s+k) ! / s !}\left|\left(R_{k} f, \pi^{k / 2} \sqrt{(s ! /(k+s) !} \mathscr{H}_{s}^{0}\right)_{\mathscr{S}, k}\right| \\
& =\pi^{-k / 2} \sqrt{(s+k) ! / s !}\left|\alpha_{s}^{k}\left(R_{k} f\right)\right| .
\end{aligned}
$$

from which it follows that $\left(\left(\mathscr{H}_{s}^{0}, f\right)\right)_{s}$ is a sequence coming from an element in $\mathscr{S}_{-k}$ iff

$$
\pi^{k} \sum_{s \in \mathbb{N}}((s+k) ! / s !)^{-1}\left|\left(\mathscr{H}_{s}^{0}, f\right)\right|^{2}<\infty
$$

Moreover, we have

$$
\begin{aligned}
\pi^{k} \sum_{s \in N}((s+k) ! / s !)^{-1}\left|\left(\mathscr{H}_{s}^{0}, f\right)\right|^{2} & =\sum_{s \in N} \mid\left(\left.\alpha_{s}^{k}\left(R_{k} f\right)\right|^{2}=\left\|R_{k} f\right\|_{\mathscr{S}, k}^{2},\right. \\
& =\|f\|_{\mathscr{S},-k}^{2},
\end{aligned}
$$


for all $f \in \mathscr{S}_{-k}$. This suggests the definition of $a_{-k}$ as the completion of the linear space of all eventually vanishing sequences $\alpha=\left(\alpha_{s}\right)_{s}$ with respect to the norm $\|\cdot\|_{\sigma_{,}-k}$ induced by the inner product

$$
(\alpha, \beta)_{\diamond,-k}=\pi^{k} \sum_{s \in N}((k+s) ! / s !)^{-1} \bar{\alpha}_{s} \beta_{s}, \quad \alpha=\left(\alpha_{s}\right)_{s}, \quad \beta=\left(\beta_{s}\right)_{s} \in \sigma_{-k} .
$$

Thus we obtain that the statements of Theorem 1 carry over to the case of general $k \in \mathbb{Z}$.

Theorem 2. The spaces $\mathscr{S}_{k}$ and $\diamond_{k}$ are unitarily equivalent for arbitrary $k \in \mathbb{Z}$. The unitary mapping $\mathscr{Y}$ between them is given by

$$
\begin{gathered}
\mathscr{Y}: \mathscr{S}_{k} \rightarrow \circlearrowleft_{k} \\
f \rightarrow\left(\left(\mathscr{H}_{s}^{0}, f\right)\right)_{s} .
\end{gathered}
$$

In particular, we have

$$
\|f\|_{\mathscr{S}, k}=\|\mathscr{Y} f\|_{o, k}=\pi^{-k / 2} \sqrt{\sum_{s \in N}((|k|+s) ! / s !)^{s g n(k)}\left|\left(\mathscr{H}_{s}^{0}, f\right)\right|^{2}}
$$

for all $f \in \mathscr{S}_{k}, k \in \mathbb{Z}$. The inverse $\mathscr{Y}^{*}$ of $\mathscr{Y}$ is given by

$$
\begin{gathered}
\mathscr{Y}^{*}: \circlearrowleft_{k} \rightarrow \mathscr{S}_{k} \\
\alpha=\left(\alpha_{s}\right)_{s} \rightarrow \sum_{s \in \mathbb{N}} \alpha_{s} \mathscr{H}_{s}^{0} .
\end{gathered}
$$

The operations $\mathscr{D}, \mathscr{F}$ and their adjoints are transferred by $\mathscr{Y}$ to corresponding operations in $\jmath_{k}, k \in \mathbb{Z}$. We have

$$
\begin{aligned}
& \mathscr{Y} \mathscr{P} \mathscr{Y}^{*} \alpha=\left(\pi^{-1 / 2} \sqrt{s} \alpha_{s-1}\right)_{s}, \quad \mathscr{Y} \mathscr{D} * \mathscr{Y}^{*} \alpha=\left(\pi^{-1 / 2} \sqrt{s+1} \alpha_{s+1}\right)_{s} \\
& \mathscr{Y} \mathscr{F} \mathscr{Y}^{*} \alpha=\left((-i)^{s} \alpha_{s}\right)_{s}, \quad \mathscr{Y} \mathscr{F} * \mathscr{Y}^{*} \alpha=\left(i^{s} \alpha_{s}\right)_{s}
\end{aligned}
$$

and in particular

$$
\mathscr{Y}\left(\mathscr{Q}^{*}\right)^{k} \mathscr{Q}^{k} \mathscr{Y}^{*} \alpha=\pi^{-k}\left(\frac{(s+k) !}{s !} \alpha_{s}\right)_{s}
$$

for all $\alpha \in \bigcup_{k \in Z} \triangleleft_{k}$. For $\left.\alpha \in\right\lrcorner_{-k}, k \in \mathbb{N}$, we have

$$
\mathscr{Y} R_{k} \mathscr{Y}^{*} \alpha=\pi^{k}\left(\frac{s !}{(s+k) !} \alpha_{s}\right)_{s}
$$


Proof. The first part of the theorem is clear from the previous considerations leading to (1.34), (1.35). It remains to establish (1.39)-(1.41) for $k \in \mathbb{Z}^{-}$. Letting $f=$ $\sum_{s \in N} \alpha_{s} \mathscr{H}_{s}^{0} \in \mathscr{S}_{-k}$ we observe that

$$
\left(f, \mathscr{Q}^{*} \mathscr{H}_{s}^{0}\right)=\left(f, \mathscr{H}_{s-1}^{0}\right) \pi^{-1 / 2} \sqrt{s}
$$

Therefore,

$$
\mathscr{Y} \mathscr{Q} f=\left(\pi^{-1 / 2} \sqrt{s} \alpha_{s-1}\right)_{s}
$$

and similarly for $\mathscr{Q}^{*}$. This proves (1.39). Because of the similarity of the proof we may now leave $(1.40)$ to the proverbial interested reader. Equations (1.41) follow directly from (1.10) and the fact that $R_{k}$ is the inverse of $\left(\mathscr{Q}^{*}\right)^{k} \mathscr{Q}^{k}$ (Lemma 1.4).

To illustrate the use of the above findings it might be entertaining to consider a few examples.

1. The exponential function $u(x)=\exp (2 \pi i z x) \gamma(x), z \in \mathbb{R}$.

We notice that $u \in L_{2}(\mathbb{R})$. In calculating the coefficients of the expansion we obtain

$$
\begin{aligned}
\left(\mathscr{H}_{s}^{0}, u\right) & =2^{1 / 4} \pi^{s / 2} / \sqrt{s !}\left(\mathscr{D}^{s} \gamma, u\right)=2^{1 / 4} \pi^{s / 2} / \sqrt{s !}\left(\gamma,\left(\mathscr{D}^{*}\right)^{s} u\right) \\
& =2^{1 / 4} \pi^{s / 2} / \sqrt{s !}\left(\gamma, \gamma D^{s} \exp (2 \pi i Z \cdot)\right) \\
& =2^{1 / 4} \pi^{s / 2} / \sqrt{s !} z^{s}\left(\gamma^{2}, \exp (2 \pi i z \cdot)\right) .
\end{aligned}
$$

The term $\left(\gamma^{2}, \exp (2 \pi i z \cdot)\right)$ is apparently the Fourier transform of $\gamma^{2}$ evaluated at $-z$, i.e. $2^{-1 / 2} \exp \left(-\pi z^{2} / 2\right)$. Thus,

$$
\left(\mathscr{H}_{s}^{0}, u\right)=2^{-1 / 4} \pi^{s / 2} / \sqrt{s !} z^{s} \exp \left(-\pi z^{2} / 2\right)
$$

and therefore

$$
\begin{aligned}
u & =2^{-1 / 4} \exp \left(-\pi z^{2} / 2\right) \sum_{s=0}^{\infty} \pi^{s / 2} / \sqrt{s !} z^{s} \mathscr{H}_{s}^{0} \\
& =\exp \left(-\pi z^{2} / 2\right) \sum_{s=0}^{\infty}(\pi z)^{s} / s ! H_{s}
\end{aligned}
$$

convergence being initially in $\mathscr{S}_{\mathbf{0}}$. Since, however, also

$$
\sum_{s=0}^{\infty}((|k|+s) ! / s !)^{s g n(k)}\left(\pi z^{2}\right)^{s} / s !=|k| ! \sum_{s=0}^{\infty}(|k|+1)^{s}\left(\pi z^{2}\right)^{s} / s !
$$




$$
=|k| ! \exp \left((|k|+1) \pi z^{2}\right)<\infty
$$

for any $k \in \mathbb{Z}$, we see that in fact $u \in \bigcap_{j \in \mathbf{Z}} \mathscr{S}_{j}$ and convergence of (1.42) is in any $\mathscr{S}_{k}, k \in \mathbb{Z}$.

\section{The Dirac $\delta$ distribution.}

The Dirac $\delta$ distribution is defined by $(\delta, \phi)=\phi(0), \phi \in \mathscr{P}_{1}$ (Note that any $\phi \in \mathscr{S}_{1}$ is continuous by the Sobolev imbedding theorem, see e.g. Theorem 4 below). Therefore its expansion with respect to Hermite functions is given by

$$
\delta=\sum_{s=0}^{\infty} \overline{\mathscr{H}_{s}^{0}(0)} \mathscr{H}_{s}^{0}=2^{1 / 2} \sum_{s=0}^{\infty} \pi^{s} / s ! \overline{H_{s}(0)} H_{s}
$$

Since $\mathscr{Q}^{2}=\mathscr{D}^{*}+2 i m \mathscr{Q}$ we obtain the following recursion formula for $H_{s}$ :

$$
H_{s+2}=\frac{s+1}{\pi} H_{s}+2 i m H_{s+1}
$$

In particular at the origin we obtain

$$
H_{s+2}(0)=\frac{s+1}{\pi} H_{s}(0)
$$

Noting that $H_{1}(0)=0, H_{0}(0)=1$, we have

$$
\begin{gathered}
H_{2 j+1}(0)=0, \quad j \in \mathbb{N}, \\
H_{2 j}(0)=(2 \pi)^{-j} \frac{(2 j) !}{j !}, \quad j \in \mathbb{N},
\end{gathered}
$$

Thus, we see that

$$
\delta=2^{1 / 2} \sum_{j=0}^{\infty}(\pi / 2)^{j} \frac{1}{j !} H_{2 j},
$$

compare [3, p. 191].

3. The sampling distribution III.

The sampling distribution III is given by

$$
(\text { III, } \phi)=\sum_{j \in Z} \phi(j) \quad \text { for } \phi \in \mathscr{S}_{3} \text {, see [6, p. 45]). }
$$

Since III is reproduced by the Fourier transform $([6$, p. 85]), i.e. 


$$
\mathscr{F} \text { III }=\text { III, }
$$

we know already by (1.37) that

$$
\left(\mathscr{H}_{4 s+t}^{0}, \text { III }\right)=\sum_{j \in \mathbf{Z}} \mathscr{H}_{4 s+t}^{0}(j)=0 \text { for } t=1,2,3 \text { and } s \in \mathbb{N} .
$$

Since $H_{s}$ is odd for $s$ odd and even for $s$ even, we are (using (1.17)) led to the interesting relation

$$
0=\sum_{j \in \mathbf{Z}} H_{4 s+2}(j)=(2 \pi)^{-(2 s+1)} \frac{(4 s+2) !}{(2 s+1) !}+2 \sum_{j \in \mathbf{Z}^{+}} H_{4 s+2}(j)
$$

or

$$
(2 \pi)^{(2 s+1)} \sum_{j \in \mathbf{Z}^{+}} H_{4 s+2}(j)=-\frac{1}{2} \frac{(4 s+2) !}{(2 s+1) !}
$$

The remaining coefficients of III are given by the obviously converging series expression

$$
\begin{aligned}
\left(\mathscr{H}_{4 s}^{0}, \text { III }\right) & =\sum_{j \in \mathbf{Z}} \mathscr{H}_{4 s}^{0}(j), \\
& =2^{1 / 4} \pi^{2 s} / \sqrt{(4 s) !} \sum_{j \in \mathbf{Z}} H_{4 s}(j) .
\end{aligned}
$$

Using the knowledge that tempered distributions are in fact elements of $\bigcup_{k \in Z} \mathscr{S}_{k}$, see [7], the last example leads to the more general question: Which tempered distributions are reproduced by the Fourier transform, i.e. are self-reciprocal, [8]? The above discussion provides a complete answer to this question in terms of Hermite expansions. In fact, we see that such distributions have to be expandable with respect to Hermite functions of order $4 j, j \in \mathbb{N}$.

Remark 3. Another way of stating the last observation is that the range of the orthogonal projection $2^{-1}(1+\mathscr{F}) \mathrm{sym}$, with sym $=2^{-1}\left(1+\mathscr{F}^{2}\right)$, i.e. the nullspace of $(\mathscr{F}-1)$ is spanned by Hermite functions of order $4 j, j \in \mathbb{N}$. The characterization to be in the range of $2^{-1}(1+\mathscr{F})$ sym is the one used in [8]. Similar results hold of course for the other 3 eigenvalues of the Fourier transform. In fact, we have that $\mathscr{F}$ has the spectral decomposition

$$
\mathscr{F}=P_{1}-i P_{-i}-P_{-1}+i P_{i}
$$

where $P_{1}=2^{-1}(1+\mathscr{F})$ sym, $\quad P_{-i}=2^{-1}(1+\mathscr{F}) \quad(1-$ sym $), \quad P_{-1}=2^{-1}(1-\mathscr{F})$ sym, $\quad P_{i}=$ $2^{-1}(1+\mathscr{F})(1-$ sym $)$ are $\left(L_{2}(\mathbb{R})\right.$-orthogonal $)$ projections on $N(\mathscr{F}-1), \quad N(\mathscr{F}+i)$, $N(\mathscr{F}+1), N(\mathscr{F}-i)$, respectively, adding up to the identity. Moreover, it is, 


$$
\begin{gathered}
\left.N(\mathscr{F}-1)=\overline{\operatorname{span}\{\mathscr{H}}{ }_{s}^{0} \mid s=4 j, j \in \mathbb{N}\right\}, \\
N(\mathscr{F}+i)=\overline{\operatorname{span}\left\{\mathscr{H}_{s}^{0} \mid s=4 j+1, j \in \mathbb{N}\right\}}, \\
N(\mathscr{F}+1)=\overline{\operatorname{span}\left\{\mathscr{H}_{s}^{0} \mid s=4 j+2, j \in \mathbb{N}\right\},} \\
N(\mathscr{F}-i)=\overline{\operatorname{span}\left\{\mathscr{H}_{s}^{0} \mid s=4 j+3, j \in \mathbb{N}\right\},}
\end{gathered}
$$

where the closure is to be taken in the sense of the underlying Sobolev type space (as introduced above) or in the sense of $\mathscr{S}=\bigcap_{k \in \mathbf{Z}} \mathscr{S}_{k}$ or $\mathscr{S}^{\prime}=\bigcup_{k \in \mathbf{Z}} \mathscr{S}_{k}$. Note that the projections are indeed acting in $\mathscr{S}_{k}, k \in \mathbb{Z}$. Since $\left(\mathscr{D}^{*}\right)^{s} \mathscr{D}^{s}$ commutes with the Fourier transform $\mathscr{F}$, it can be seen that orthogonality is also preserved with respect to all these spaces.

We shall conclude this presentation by a brief discussion of the generalization to higher dimensions.

\section{Extension to the higher-dimensional case}

The results of the previous section can be easily extended to the higher-dimensional case by adopting a suitable multi-index and multi-argument notation. The tempered derivative and its adjoint have now to be interpreted as partial derivatives.

The Gaussian distribution function now goes over into

$$
\gamma(x)=e^{-\pi x^{2}}, \quad x \in \mathbb{R}^{n}, \quad n \text { a fixed positive integer, }
$$

where $x^{2}=x \cdot x$ in the sense of the Cartesian inner product of $\mathbb{R}^{n}$.

For $\Phi \in \mathscr{C}_{\infty}\left(\mathbb{R}^{n}\right)\left(\check{C}_{\infty}\left(\mathbb{R}^{n}\right)\right.$ denotes the set of arbitrarily often continuously differentiable (complex-valued) functions with compact support in $\mathbb{R}^{n}$ ) we define now

$$
\mathscr{Q}_{k} \Phi:=\left(\gamma^{-1} D_{k} \gamma\right) \Phi \text { for } \Phi \in \mathcal{C}_{\infty}\left(\mathbb{R}^{n}\right)
$$

Here

$$
\begin{gathered}
\gamma^{-1}(x):=1 / \gamma(x), \quad x \in \mathbb{R}^{n}, \\
D_{k} \Phi=(2 \pi i)^{-1} \partial_{k} \Phi \text { for } \Phi \in \mathcal{C}_{\infty}\left(\mathbb{R}^{n}\right),
\end{gathered}
$$

where the " $\partial_{k}$ " indicates ordinary partial differentiation with respect to the $k$ th argument. Denoting the Fourier transform again by $\mathscr{F}$ with the convention that

$$
(\mathscr{F} \Phi)(x)=\int_{\mathbb{R}^{n}} e^{-2 \pi i x \cdot 1} \Phi(t) \mathrm{dt} \text { for all } \Phi \in \mathcal{C}_{\infty}\left(\mathbb{R}^{n}\right)
$$

we have that $\mathscr{F}$ is a spectral representation for the family of commuting operators 
$\left(\underline{D}_{k}\right)_{k=1, \ldots n}$, where $\underline{D}_{k}$ denotes the closure of $D_{k}$. Denoting the self-adjoint extension of the "multiplication-by-the $k$ th-argument" operator by $m_{k}$ we may express this by

$$
\mathscr{F} \underline{D}_{k}=\underline{m}_{k} \mathscr{F} \quad \text { on } \quad \mathcal{C}_{\infty}\left(\mathbb{R}^{n}\right), \quad k=1,2, \ldots, n .
$$

The relationship between $\mathscr{F}$ and $\mathscr{D}, \mathscr{Q}^{*}$ carry over almost literally from the onedimensional case. The announced multi-index notation is introduced by letting

$$
\begin{gathered}
\mathscr{Q}^{s}=\mathscr{Q}_{1}^{s_{1}} \mathscr{Q}_{2}^{s_{2}} \ldots \mathscr{Q}_{n}^{s_{n}}, \\
\left.\left(\mathscr{Q}^{*}\right)^{s}=(\mathscr{\mathscr { Q }})^{*}\right)^{s_{1}}\left(\mathscr{Q}_{2}^{*}\right)^{s_{2}} \ldots\left(\mathscr{Q}_{n}^{*}\right)^{s_{n}} .
\end{gathered}
$$

and

$$
\mathscr{H}_{s}^{0}(x)=\mathscr{H}_{s_{1}}^{0}\left(x_{1}\right) \mathscr{H}_{s_{2}}^{0}\left(x_{2}\right) \ldots \mathscr{H}_{s_{n}}^{0}\left(x_{n}\right)
$$

where $s=\left(s_{1}, s_{2}, \ldots, s_{n}\right) \in \mathbb{N}^{n}$ and $x=\left(x_{1}, x_{2}, \ldots, x_{n}\right) \in \mathbb{R}^{n}$. In the following we also shall make use of the following multi-index conventions:

$$
\begin{gathered}
s \leqq t \text { iff } s_{k} \leqq t_{k} \text { for } k=1,2, \ldots, n . \\
s+t=r \text { iff } s_{k}+t_{k}=r_{k} \text { for } k=1,2, \ldots, n, s, t \in \mathbb{Z}^{n}, \\
s t=r \text { iff } s_{k} t_{k}=r_{k} \text { for } k=1,2, \ldots, n, s, t \in \mathbb{Z}^{n}, \\
s !=s_{1} ! s_{2} ! \ldots s_{n} !, \quad s^{t}=s_{1}^{t_{1}} s_{2}^{t_{2}} \ldots s_{n}^{t_{n}}, \\
1 / s !=1 / s ! 1 s_{2} ! \ldots 1 / s_{n} ! \text { for } \quad s \in \mathbb{N}^{n}, t \in \mathbb{Z}^{n},
\end{gathered}
$$

and

$$
\begin{gathered}
\Sigma s=\sum_{k=1}^{n} s_{k} \text { for } s \in \mathbb{Z}^{n}, \\
|s|=\left(\left|s_{1}\right|,\left|s_{2}\right|, \ldots,\left|s_{n}\right|\right) \\
\operatorname{sgn}(s)=\left(\operatorname{sgn}\left(s_{1}\right), \operatorname{sgn}\left(s_{2}\right), \ldots, \operatorname{sgn}\left(s_{n}\right)\right) .
\end{gathered}
$$

To simplify formulae we shall write $a^{s}$ for $a^{\Sigma_{s}} \equiv a^{s_{1}} a^{s_{2}} \ldots a^{s_{n}}$, and use occasionally $\emptyset, I$ meaning the multi-index $(0,0, \ldots, 0)$ and $(1,1, \ldots, 1)$, respectively.

We proceed by defining Sobolev type spaces $\mathscr{S}_{s}$ for $s \in \mathbb{Z}^{n}$ as the tensor product Hilbert space

$$
\mathscr{S}_{s}=\mathscr{S}_{s_{1}} \otimes \mathscr{S}_{s_{2}} \otimes \cdots \otimes \mathscr{S}_{s_{n}}
$$


with inner product $(\cdot, \cdot)_{\mathscr{S}, s}$ induced by the product of the inner products of the factor spaces. The corresponding norm will be denoted by $\|\cdot\|_{\mathscr{Y}_{. s}}$. The transition to these spaces lead to a straightforward generalization of the previous considerations. In particular, if $s \geqq \emptyset$ then we have

$$
(\cdot, \cdot)_{\mathscr{S}, s}=\left(\mathscr{D}^{s}, \mathscr{D}^{s} \cdot\right)
$$

We shall also consider Hilbert spaces $\mathscr{S}_{k}, k \in \mathbb{Z}$, defined by

$$
\mathscr{T}_{k}=\bigcap_{\substack{\Sigma s=|k| \\ s \geqq \emptyset}} \mathscr{S}_{\mathrm{sgn}(k) s}, \quad k \in \mathbb{Z},
$$

with norm $\|\cdot\|_{\mathscr{S}, k}$ induced by the inner product

$$
(\cdot, \cdot)_{\mathscr{S}, k}=\sum_{\substack{\boldsymbol{\Sigma} s=|\boldsymbol{k}| \\ s \geqq \emptyset}}(\cdot, \cdot)_{\mathscr{S}, \mathrm{sgn}(\mathrm{k}) \mathrm{s}} \cdot
$$

In particular we have

$$
\mathscr{S}_{0}=\mathscr{S}_{\varphi}=L_{2}\left(\mathbb{R}^{n}\right)
$$

The next lemma summarizes some of the analogues to the results in the previous section:

Lemma 2.1. Let $p, m \in \mathbb{Z}$ (or $p, m \in \mathbb{Z}^{n}$ ) and $p \leqq m$ then we have that $\mathscr{S}_{m} \hookrightarrow \mathscr{S}_{p}$. In particular, we have

$$
\|\Phi\|_{\mathscr{S}, p}^{2} \leqq(2 \pi)^{m-p}\|\Phi\|_{\mathscr{S}^{\prime}, m}^{2} \quad \text { for } \quad \Phi \in \mathscr{S}_{m}
$$

Moreover, the Hermite functions $\mathscr{H}_{s}^{0}, s \in \mathbb{N}^{n}$, form a complete, orthogonal family of eigenfunctions of $\left(\mathscr{Q}^{*}\right)^{t} \mathscr{D}^{t}$ and $\mathscr{F}, t \in \mathbb{N}^{n}$, in any of the spaces $\mathscr{S}_{r} r \in \mathbb{Z}$ (or $\left.r \in \mathbb{Z}^{n}\right)$, and we have

$$
\begin{gathered}
\left(\mathscr{Q}^{*}\right)^{t} \mathscr{Q}^{t} \mathscr{H}_{s}^{0}=\frac{(s+t) !}{s ! \pi^{t}} \mathscr{H}_{s}^{0}, \\
\mathscr{F} \mathscr{H}_{s}^{0}=(-i)^{s} \mathscr{H}_{s}^{0}, \\
\left(\mathscr{D}^{*}\right)^{t} \mathscr{H}_{s}^{0}=\pi^{-t / 2} \sqrt{s ! /(s-t) !} \mathscr{H}_{s-t}^{0} \quad \text { for } s \geqq t \\
\mathscr{Q}^{t} \mathscr{H}_{s}^{0}=\pi^{-t / 2} \sqrt{(s+t) ! / s !} \mathscr{H}_{s+t}^{0},
\end{gathered}
$$

for all $s, t \in \mathbb{N}^{n}$. 
Proof. The results of this lemma are easily obtained as straightforward transcriptions of the one-dimensional proofs into multi-index notation. For sake of brevity we shall not give the details.

We need to define suitable sequence spaces $\lrcorner_{k}$ and $\jmath_{s}, k \in \mathbb{Z}, s \in \mathbb{Z}^{n}$. The sequences are now conveniently enumerated using non-negative multi-indices, i.e. elements of $\mathbb{N}^{n}$. The space $\sigma_{s}$ may be introduced as the completion of eventually vanishing sequences $\left(\alpha_{t}\right)_{t}$ with respect to the norm $\|\cdot\|_{0 . s}$ induced by the inner product

$$
(\alpha, \beta)_{o, s}=\pi^{-s} \sum_{t \in N^{n}}((|s|+t) ! / t !)^{\operatorname{sgn}(s)} \bar{\alpha}_{t} \beta_{t}
$$

with $\alpha=\left(\alpha_{t}\right)_{t}, \beta=\left(\beta_{t}\right)_{t} \in \diamond_{s}$. The appropriate spaces $\diamond_{k}, k \in \mathbb{Z}$, can be defined as the Hilbert space of sequences

$$
\alpha=\left(\alpha_{t}\right)_{t} \in \bigcap_{\substack{\Sigma s=|k| \\ s \geqq \emptyset}} J_{s g n(k) s}
$$

with inner product

$$
(\alpha, \beta)_{\diamond, k}=\pi^{-k} \sum_{t \in N^{n}}\left[\sum_{\substack{\Sigma s=|k| \\ s \geqq 0}}(s+t) ! / t !\right]^{s g n(k)} \bar{\alpha}_{t} \beta_{t}
$$

with $\left.\alpha=\left(\alpha_{t}\right)_{t}, \beta=\left(\beta_{t}\right)_{t} \in\right\lrcorner_{k}$. Clearly we have that the spaces $\lrcorner_{k}, \diamond_{s}, k \in \mathbb{Z}, s \in \mathbb{Z}^{n}$, are Hilbert spaces.

With the above definitions we are now ready to formulate the higher-dimensional analogue of Theorem 2 .

Theorem 3. The spaces $\mathscr{S}_{s}$ and $o_{s}$ are unitarily equivalent for arbitrary $s \in \mathbb{Z}^{n}$. The unitary mapping $\mathscr{Y}$ between them is given by

$$
\begin{gathered}
\mathscr{Y}: \mathscr{S}_{s} \rightarrow \sigma_{s} \\
f \rightarrow\left(\left(\mathscr{H}_{t}^{0}, f\right)\right)_{t} .
\end{gathered}
$$

In particular, we have

$$
\|f\|_{\mathscr{S}, s}=\|\mathscr{Y} f\|_{o, s}=\pi^{-s / 2} \sqrt{\sum_{t \in \mathrm{N}^{n}}((|s|+t) ! / t !)^{s g n(s)}\left|\left(\mathscr{H}_{t}^{0}, f\right)\right|^{2}}
$$

for all $f \in \mathscr{S}_{s}, s \in \mathbb{Z}^{n}$. The inverse $\mathscr{Y}^{*}$ of $\mathscr{Y}$ is given by

$$
\mathscr{Y}^{*}: \jmath_{s} \rightarrow \mathscr{I}_{s}
$$




$$
\alpha=\left(\alpha_{t}\right)_{t} \rightarrow \sum_{t \in N^{n}} \alpha_{t} \mathscr{H}_{t}^{0}
$$

By the same association $\mathscr{Y}$ now considered as a mapping between $\mathscr{S}_{k}$ and $\jmath_{k}, k \in \mathbb{Z}, a$ unitary equivalence is furnished between these spaces. The isometry is given explicitly by

$$
\|f\|_{\mathscr{S}, k}=\left\|\mathscr{Y}_{f}\right\|_{0, k}=\pi^{-k / 2} \sqrt{\sum_{i \in N^{n}}\left[\sum_{\substack{\Sigma s=|k| \\ s \geqq \emptyset}}(s+t) ! / t !\right]^{\operatorname{sgn}(k)}\left|\left(\mathscr{H}_{t}^{0}, f\right)\right|^{2}},
$$

for all $f \in \mathscr{S}_{k}, k \in \mathbb{Z}$. The operations $\mathscr{D}, \mathscr{F}$ and their adjoints are transferred by $\mathscr{Y}$ to corresponding operations in $s_{s}, s \in \mathbb{Z}^{n}$, and $s_{k}, k \in \mathbb{Z}$. We have $\left(\alpha_{s} \equiv 0\right.$ if not $\left.s \geqq \emptyset\right)$

$$
\begin{gathered}
\mathscr{Y} \mathscr{D}^{r} \mathscr{Y}^{*} \alpha=\left(\pi^{-r / 2} \sqrt{t ! /(t-r) !} \alpha_{t-r}\right)_{t}, \\
\mathscr{Y}\left(\mathscr{D}^{*}\right)^{r \mathscr{Y}}{ }^{*} \alpha=\left(\pi^{-r / 2} \sqrt{(t+r) / t !} \alpha_{t+r}\right)_{t}, \\
\mathscr{Y} \mathscr{F} \mathscr{Y}^{*} \alpha=\left((-i)^{t} \alpha_{t}\right)_{t}, \quad \mathscr{Y} \mathscr{F} * \mathscr{Y}^{*} \alpha=\left(i^{t} \alpha_{t}\right)_{t}, \\
\mathscr{Y}\left(\mathscr{Q}^{*}\right)^{s} \mathscr{D}^{s} \mathscr{Y}^{*} \alpha=\pi^{-s}\left(\frac{(s+t) !}{t !} \alpha_{t}\right)_{t},
\end{gathered}
$$

for all $\alpha \in \bigcup_{s \in Z^{n} \jmath_{s}}=\bigcup_{k \in Z} \circlearrowleft_{k}$. In particular for $\alpha \in \jmath_{-s}, s \in \mathbb{Z}^{n}, s \geqq \emptyset$, we have

$$
\mathscr{Y} R_{s} \mathscr{Y}^{*} \alpha=\pi^{s}\left(\frac{t !}{(s+t) !} \alpha_{t}\right)_{t}
$$

where $R_{s}$ denotes the Riesz map associated with $\mathscr{S}_{-s}$, and similarly for $\left.\alpha \in\right\lrcorner_{-k}, k \in \mathbb{N}$,

$$
\mathscr{Y} R_{k} \mathscr{Y}^{*} \alpha=\pi^{k}\left(\left[\sum_{\substack{\Sigma s=k \\ s \geqq \emptyset}}(s+t) ! / t !\right]^{-1} \alpha_{t}\right)^{t}
$$

where $R_{k}$ denotes the Riesz map associated with $\mathscr{S}_{k}$.

Proof. The theorem for $\mathscr{S}_{s}$-spaces, $s \in \mathbb{Z}^{n}$, follows by merely transcribing the arguments used in Theorem 2 into multi-index notation. The result can also be seen in this case directly from the tensor product structure of $\mathscr{S}_{s}$ and $\sigma_{s}, s \in \mathbb{Z}^{n}$. Formulae (2.16)-(2.18) remain unchanged if we discuss the spaces $\mathscr{P}_{k}$ and $o_{k}$ with $k \in \mathbb{Z}$. As in Theorem 1 it follows that $\mathscr{Y}$ is also onto in both cases. The rest of the theorem can be obtained as in the one-dimensional case using the analogous multi-index results compiled in the previous lemma. Note in particular that $(k \in \mathbb{N})$ 


$$
\mathscr{H}_{t}^{0}=\pi^{k}\left[\sum_{\substack{\sum_{s=k} \\ s \geqq \emptyset}}(s+t) ! / t !\right]^{-1} \sum_{\substack{\sum s=k \\ s \geqq \emptyset}}\left(\mathscr{Q}^{*}\right)^{s} \mathscr{Q}^{s} \mathscr{H}_{t}^{0}
$$

\section{Some consequences}

As an interesting application of the results of Chapter 2 we record in our final chapter some easily established imbedding properties.

Theorem 4. For the spaces $\mathscr{S}_{k}, \diamond_{k}$ and $\mathscr{S}_{s}, \jmath_{s} . k \in \mathbb{Z}, s \in \mathbb{Z}^{n}$, we have the following imbedding results

(a) $\mathscr{S}_{k I} \hookrightarrow \mathscr{S}_{k} \hookrightarrow \mathscr{S}_{[k / n] I}, J_{k I} \hookrightarrow J_{k} \hookrightarrow \sigma_{[k / n] I}$ for $k \geqq 0$,

$$
\mathscr{S}_{[k / n] I} \hookrightarrow \mathscr{S}_{k} \hookrightarrow \mathscr{S}_{k I}, \supset_{[k / n] I} \hookrightarrow \supset_{k} \hookrightarrow \supset_{k I} \text { for } k \leqq 0
$$

and

(b) the imbeddings $\mathscr{S}_{k+1} \hookrightarrow \mathscr{S}_{k}, \mathscr{S}_{s+1} \hookrightarrow \mathscr{S}_{s}, J_{k+1} \hookrightarrow J_{k}, J_{s+1} \hookrightarrow J_{s}$, are compact, i.e.

$$
\begin{gathered}
\mathscr{S}_{k+1} \hookrightarrow \hookrightarrow \mathscr{S}_{k}, \quad \mathscr{S}_{s+I} \hookrightarrow \hookrightarrow \mathscr{S}_{s}, \\
J_{k+1} \hookrightarrow \hookrightarrow J_{k}, \quad J_{s+I} \hookrightarrow \hookrightarrow J_{s} .
\end{gathered}
$$

Proof. We first notice that it suffices to show the imbedding results for the sequence spaces. The remaining results follow via the use of $\mathscr{Y}$. For sake of brevity we only consider the case $k \geqq 0$, the other case being analogous. It is clear that for any $s \geqq \emptyset$, $\Sigma s \leqq k$,

$$
\pi^{-k / 2} \sqrt{\sum_{t \in N^{n}}(s+t) ! / t !\left|\alpha_{t}\right|^{2}} \leqq \pi^{-k / 2} \sqrt{\sum_{t \in N^{n}}\left[\sum_{\substack{\sum_{r}=k \\ r \geqq \emptyset}}(r+t) ! / t !\right]\left|\alpha_{t}\right|^{2}},
$$

where $\alpha=\left(\alpha_{t}\right)_{t} \in \sigma_{k}$. Now replacing $s$ by a multi-index with equal entries given by the integer value $[k / n]$ of $k / n$, i.e. $s=[k / n] I$, we obtain

$$
\pi^{-(k-n[k / n]) / 2} \pi^{-s / 2} \sqrt{\sum_{t \in N^{n}}(s+t) ! / t !\left|\alpha_{t}\right|^{2}} \leqq \pi^{-k / 2} \sqrt{\sum_{t \in N^{n}}\left[\sum_{\substack{i r=k \\ r \geqq \emptyset}}(r+t) ! / t !\right]\left|\alpha_{t}\right|^{2}}
$$

which in turn proves

$$
J_{k} \hookrightarrow J_{[k / n] I}
$$


The remaining imbedding follows since for $s=k I$ we have

$$
\pi^{-k} \sum_{t \in \mathbb{N}^{n}}\left[\sum_{\substack{\sum_{r=k}=\phi \\ r \geqq \emptyset}}(r+t) ! / t !\right]\left|\alpha_{t}\right|^{2} \leqq k^{n} \pi^{k(n-1)} \pi^{-k n} \sum_{t \in \mathbb{N}^{n}}(s+t) ! / t !\left|\alpha_{t}\right|^{2}
$$

To show (b) let $\left(\alpha^{m}\right)_{m \in N}, \alpha^{m}=\left(\alpha_{s}^{m}\right)_{s} \in J_{k+1}$, be a uniformly bounded sequence in $\diamond_{k+1}$ i.e.

$$
\left\|\alpha^{m}\right\|_{0, k+1} \leqq C
$$

for all $m \in \mathbb{N}$ and some $C>0$. Now,

$$
\left\|\alpha^{m}\right\|_{0, k}^{2} \leqq \pi^{-k} \sum_{\substack{t \in N^{n} \\ \Sigma t \leqq M}}\left[\sum_{\substack{\Sigma r=k \\ r \geqq \emptyset}}(r+t) ! / t !\right]\left|\alpha_{t}^{m}\right|^{2}+\pi^{-k} \sum_{\substack{t \in N^{n} \\ \Sigma}>M}\left[\sum_{\substack{\sum_{r}=k \\ r \geqq \emptyset}}(r+t) ! / t !\right]\left|\alpha_{t}^{m}\right|^{2}
$$

The second term on the right-hand side can be estimated uniformly w.r.t. $m \in \mathbb{N}$ by

$$
\pi^{-k} M^{-1} \sum_{\substack{t \in N^{n} \\ \Sigma t>M}}\left[\sum_{\substack{\Sigma \\ r=k+1 \\ r \geqq \emptyset}}(r+t) ! / t !\right]\left|\alpha_{t}^{m}\right|^{2} \leqq \pi M^{-1} C
$$

since

$$
\begin{aligned}
& {\left[\sum_{\substack{\Sigma_{r}=k \\
r \geqq \emptyset}}(r+t) ! / t !\right]\left[\sum_{\substack{\Sigma_{r}=k+1 \\
r \geqq \emptyset}}(r+t) ! / t !\right]^{-1} \leqq\left[\sum_{\substack{\Sigma r=k \\
r \geqq \emptyset}}(r+t) ! / t !\right]\left[\sum_{\substack{\Sigma \delta=1 \\
\delta \geqq \emptyset}} \sum_{\substack{\Sigma r=k \\
r \geqq \emptyset}}(r+\delta+t) ! / t !\right]^{-1}} \\
& \leqq\left[\sum_{\substack{\Sigma_{r=k}=k \\
r \geqq \emptyset}}(r+t) ! / t !\right]\left[\sum_{j=1}^{n} \sum_{\substack{\sum_{r}=k \\
r \geqq \emptyset}}\left(r_{j}+1+t_{j}\right)(r+t) ! / t !\right]^{-1} \leqq\left[\sum_{j=1}^{n} t_{j}\right]^{-1} \leqq M^{-1} .
\end{aligned}
$$

On the basis of this uniform estimate the existence of a convergent subsequence of $\left(\alpha^{m}\right)_{m}$ in $s_{k}$ can be shown by a standard diagonal argument choosing consecutive subsequences.

The compactness of the imbedding $\partial_{s+1} \hookrightarrow \hookrightarrow \partial_{s}$ follows by similar arguments.

Remark 4. The compact imbedding property stated as part (b) of the previous theorem has been shown for the two types of $\mathscr{S}$-spaces in [7]. The above considerations provide an independent proof of this important result.

Given that the tempered distributions $\mathscr{S}^{\prime}$ can in fact be identified as elements of $\bigcup_{k \in \mathbf{Z}} \mathscr{S}_{k}$, [7], with the natural induced convergence concept (inductive limit), it can be seen from the imbedding results of the above theorem that

$$
\mathscr{S}^{\prime}=\bigcup_{k \in \mathbf{Z}} \mathscr{S}_{k}=\bigcup_{s \in \mathbf{Z}^{n}} \mathscr{S}_{s}
$$


In fact, it would be sufficient (according to Theorem 4) to choose any sequence of indices $k \rightarrow-\infty$ or multi-indices $s \rightarrow(-\infty,-\infty, \ldots,-\infty)$ to perform the union. In particular, we have the characterization of tempered distributions given in our final theorem.

Theorem 5. Any tempered distribution can be expanded with respect to Hermite functions in a series converging in $\mathscr{S}_{k}$ for some $k \in \mathbb{Z}$ (or in $\mathscr{S}_{s}$ for some $s \in \mathbb{Z}^{n}$ ). We have

$$
\begin{gathered}
f \in \mathscr{S}^{\prime} \quad \text { iff } \sum_{t \in N^{n}}\left[\sum_{\substack{\Sigma s=|k| \\
s \geqq \emptyset}}(s+t) ! / t !\right]^{\mathrm{sgn}(k)}\left|\left(\mathscr{H}_{t}^{0}, f\right)\right|^{2}<\infty \text { for some } k \in \mathbb{Z}, \\
\text { iff } \sum_{t \in N^{n}}((|s|+t) ! / t !)^{\operatorname{sgn}(s)}\left|\left(\mathscr{H}_{t}^{0}, f\right)\right|^{2}<\infty \text { for some } s \in \mathbb{Z}^{n} .
\end{gathered}
$$

Proof. The result is clear from the above admissions on the relationship between the tempered distributions and $\mathscr{S}$-spaces.

Remark 5. The last theorem reproduces in the context developed here a result previously obtained in [3, Chapter 8]. The difference is that the concept of convergence is in our approach specified as a particular Sobolev space convergence. The additional structure provided by this allows for an isometric control over the type of convergence in the sequence spaces.

\section{REFERENCES}

1. N. I. Axhiezer and I. M. Glasman, Theory of Linear Operators in Hilbert Space, Vol. 1 (Pitman Publ. Ltd., London, 1981).

2. N. I. Akhiezer and I. M. Glasman, Theory of Linear Operators in Hilbert Space, Vol. 2 (Pitman Publ. Ltd., London, 1981).

3. P. Antosik, J. Mikusinski and R. Sikorski, Theory of Distributions, The Sequential Approach (Elsevier Scientific Publ. Co., New York, 1973).

4. G. Bachman and L. Narici, Functional Analysis (Academic Press, New York, 1966).

5. R. A. Bonic, Linear Functional Analysis (Gordon and Breach, New York, 1969).

6. R. Picard, A Hilbert Space Approach to Some Classical Integral Transforms (Pitman Research Notes in Mathematics, Longman, New York, 1989).

7. R. Picard, A Hilbert space approach to distributions. Proc. Roy. Soc. Edinburgh 115A (1990), 275-288.

8. E. C. Titchmarsh, Introduction to the Theory of Fourier Integrals (Clarendon Press, Oxford, 1948).

Department of Mathematical Sciences

University OF Wisconsin-MilwaUKEE

MilwaukeE, Wisconsin 53211, U.S.A. 